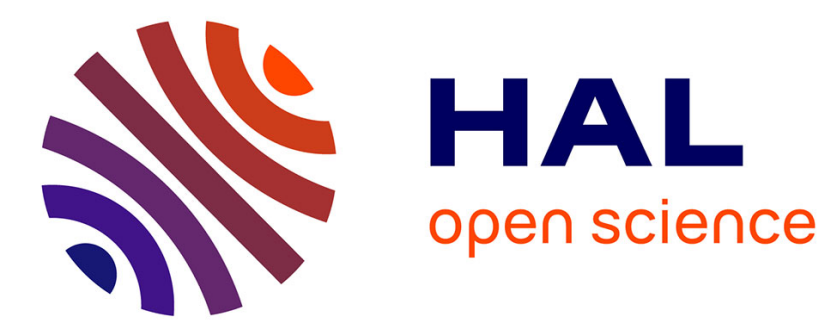

\title{
An approach to reliability-based shape and topology optimization of truss structures
}

André Jacomel Torii, Rafael Holdorf Lopez, Fabio Biondini

\section{To cite this version:}

André Jacomel Torii, Rafael Holdorf Lopez, Fabio Biondini. An approach to reliability-based shape and topology optimization of truss structures. Engineering Optimization, 2011, pp.1. 10.1080/0305215X.2011.558578 . hal-00712746

\section{HAL Id: hal-00712746 \\ https://hal.science/hal-00712746}

Submitted on 28 Jun 2012

HAL is a multi-disciplinary open access archive for the deposit and dissemination of scientific research documents, whether they are published or not. The documents may come from teaching and research institutions in France or abroad, or from public or private research centers.
L'archive ouverte pluridisciplinaire HAL, est destinée au dépôt et à la diffusion de documents scientifiques de niveau recherche, publiés ou non, émanant des établissements d'enseignement et de recherche français ou étrangers, des laboratoires publics ou privés. 


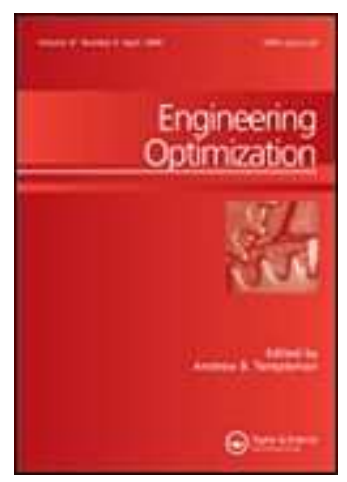

\section{An approach to reliability-based shape and topology optimization of truss structures}

\begin{tabular}{|r|l|}
\hline Journal: & Engineering Optimization \\
\hline Manuscript ID: & GENO-2010-0171.R1 \\
\hline Manuscript Type: & Original Article \\
\hline Date Submitted by the \\
Author: & $15-$ Nov-2010 \\
\hline Keywords: List of Authors: & $\begin{array}{l}\text { Torii, André; Universidade Federal do Paraná, CESEC } \\
\text { Lopez, Rafael; Institut National des Sciences Appliquees de Rouen, } \\
\text { Laboratoire Mecanique de Rouen (LMR) } \\
\text { Biondini, Fabio; Politecnico di Milano, Dipartimento di Ingegneria } \\
\text { Strutturale }\end{array}$ \\
\hline & \\
\hline
\end{tabular}

\section{SCHOLARONE $^{\text {m }}$ Manuscripts}




\title{
An approach to reliability-based shape and topology optimization of truss structures
}

\author{
André Jacomel Torii ${ }^{1}$, Rafael Holdorf Lopez ${ }^{2 *}$, Fabio Biondini $^{3}$ \\ ${ }^{1}$ Centro de Estudos de Engenharia Civil (CESEC), Universidade Federal do Paraná, \\ Curitiba, Brazil \\ ${ }^{2}$ Laboratoire de Mécanique de Rouen, Institut National des Sciences Appliquées \\ (INSA) de Rouen, France \\ ${ }^{3}$ Dipartimento di Ingegneria Strutturale (DIS), Politecnico di Milano, Italy
}

*Corresponding author: rafaelholdorf@gmail.com.

(Received 9 August 2010; final version received 15 November 2010)

\begin{abstract}
This article deals with the reliability based geometry and topology optimization of truss structures. It presents an approach to optimize simultaneously the geometry and topology of statically undetermined trusses considering the acting forces and the yielding stress of the bars as random variables. Based on the assumptions of linear structural behaviour and independent and normally distributed random variables, the optimization problem is posed in such a way that its computational cost is similar to a standard deterministic optimization problem, which is the main contribution of this article. It is shown in the numerical analysis that when uncertainties are considered, the resulting optimum structure may not be a simply scaled version of the deterministic solution, but there may be a change in the structural geometry as well.
\end{abstract}

Keywords: reliability based optimization; truss structures; geometry optimization; topology optimization

\section{Introduction}

Methods for the optimization of trusses, where the members areas are taken as design variable, are well established and there is a rich literature on this subject (Hemp, 1973; Pedersen, 1970; Pedersen, 1993). The problem of truss shape optimization, where the nodes' positions are taken as design variables, is also addressed in literature (Achtziger, 2006; Achtziger, 2007; Kocvara and Zowe, 1996), but not as extensively. One of the main reasons why sizing optimization was studied more frequently than shape optimization, also called here geometry optimization, is that the sizing optimization problem can, in some cases, be stated as a linear programming problem. For such cases, very efficient methods are available and it is possible to guarantee certain important mathematical properties of the solution, such as the existence of a 
global optimum (Nocedal and Wright, 1999; Rao, 1996; Arora, 2004). The truss geometry optimization problem is, instead, nonlinear by its nature, and therefore it needs to be solved by nonlinear optimization methods, which are in general more complex and computationally demanding than the linear programming techniques. Also, the geometry optimization of a truss may lead to a non-convex optimization problem, and several local minima may exist (Achtziger 2006; Achtziger 2007). In the last decades the increase in computational resources allowed an increase in the use of nonlinear optimization methods, and thus the problem of geometry optimization was studied more extensively. Some strategies have been developed to deal with such optimization problem, and with respect to Achtziger (2007) these strategies can be grouped as: simultaneous optimization of truss topology and geometry, alternating optimization and implicit programming optimization.

In deterministic optimization, however, the uncertainties involved in the design problem, such as those affecting material properties and loads, among others, are not considered. Robust optimization or reliability based design optimization (RBDO) methods are usually employed to take such uncertainties into account (see, for instance, Beyer and Sendhoff (2006), Schuëller and Jensen (2009)). The former has as main goal the minimization of the variability of some parameters related to system response due to its uncertainties. For example, Calafiore and Dabbene (2008) applied this concept in the field of design of truss structures.

The main goal of the RBDO is to optimize a structure and guarantee that its probability of failure is lower than a certain level chosen a priori by the designer. Nakib (1991), Thampan and Krishnamoorty (2001), Togan and Daloglu (2006) dealt with the RBDO of truss structures, regarding only the size optimization of such 
structures. Lagaros et al. (2007) pursued the reliability based robust optimization of trusses grouping at the same time the goals of the robust and RBDO.

However, only a few articles have dealt with the reliability based shape and topology optimization of truss structures (Morutsu and Shao, 1990; Stocki et al., 2001). Thus, this article proposes a probabilistic approach for solving the simultaneous optimization of geometry and topology for statically indeterminate trusses, taking into account the uncertainties on the applied forces as well as the yielding stresses. In this approach the applied forces and the yielding stresses are modeled as random variables, and the failure constraints are expressed in probabilistic terms.

This study has three main contributions: (i) It is shown how to efficiently pursue the sensitivity analysis required by the gradient based method used in the optimization process; (ii) Based on the assumptions of linear structural behavior and independent and normally distributed random variables, the RBDO problem is posed in such a way that the reliability of the structure is accessed directly, without using iterative methods such as a First Order Reliability Method (FORM) or Monte Carlo Simulation (MCS) (Haldar and Mahadevan, 2000).; (iii) It is shown that, when uncertainties are considered, the resulting optimum structure may not be a simply scaled version of the deterministic solution (e.g. higher cross-section areas), but there may be a change in the structural geometry as well.

\section{Deterministic optimization}

Deterministic optimization of geometry and topology of trusses is presented in the following, with emphasis on how the sensitivities may be efficiently evaluated. 


\subsection{Formulation of the optimization problem}

The optimization problem is posed as the minimization of the volume of the structure subject to stress constraints by taking the nodal coordinates and cross-section areas as design variables:

Find: $\mathbf{x}$ and $\mathbf{A}$

that gives

$$
\min V(\mathbf{x}, \mathbf{A})=\mathbf{A}^{T} \cdot \mathbf{L}(\mathbf{x})
$$

subject to

$$
\begin{aligned}
& g_{j}=+\sigma_{j}-\sigma_{t} \leq 0 \quad(j=1,2, \ldots, m) \\
& g_{j+m}=-\sigma_{j}+\sigma_{c} \leq 0 \quad(j=1,2, \ldots, m)
\end{aligned}
$$

where $V$ is the volume of the structure, $\mathbf{x}$ is the vector of nodal coordinates, $\mathbf{A}$ is the vector of member areas, $\mathbf{L}$ is the vector of member lengths, $g_{j}$ are stress constraints, $\sigma_{j}$ is the stress on member $j, \sigma_{t}$ is the yielding stress in tension, $\sigma_{c}$ is the yielding stress in compression and $m$ is the number of members subjected to stress constraints. In this article, buckling constraints are not introduced.

For convenience, the design variables $\mathbf{A}$ and $\mathbf{x}$ can be grouped into a single design vector $\mathbf{X}$, and the constraints from Eq. (2) and Eq. (3) can be grouped into a single vector of constraints $\mathbf{g}$. In this way, the previous problem is rewritten as follows:

\section{Find: $\mathbf{X}$}

that gives

$$
\min V(\mathbf{X})=\mathbf{A}^{T} \cdot \mathbf{L}
$$

subject to

$$
\mathbf{g} \leq \mathbf{0},
$$


where $\mathbf{g}$ is a vector with $2 m$ components since there are two constraints defined for each bar of the structure.

\subsection{Sensitivity analysis}

Most nonlinear optimization methods need to evaluate the gradients of both the objective function and constraints with respect to the design variables. The partial derivative of the volume $V$ with respect to each member area is given by the length of each bar. The partial derivative of $V$ with respect to the nodal coordinates is also easy to obtain, and expressions similar to the ones presented by Martínez et al. (2007) and Torii and Biondini (2009) can be used for this purpose. Moreover, all these derivatives can be efficiently evaluated by finite differences, if necessary, since the evaluation of $V$ is not computationally demanding.

The major difficulty arises when evaluating the gradients of the constraints from Eq. (2) and Eq. (3), since these constraints involve stresses that depend implicitly on nodal displacements. From Eq. (2) and Eq. (3) it can be seen that the partial derivatives from these expressions will be the same with opposite sign and, consequently, only one of them must be evaluated numerically.

Working with an arbitrary chosen constraint $g_{j}$, as defined in Eq. (2), its partial derivative with respect to a design variable $X_{i}$ can be written as

$$
\frac{\partial g_{j}}{\partial X_{i}}=\frac{d \sigma_{j}}{d X_{i}}+\frac{\partial \sigma_{j}^{T}}{\partial \mathbf{u}} \cdot \frac{\partial \mathbf{u}}{\partial X_{i}}
$$

where the symbol $d$ stands for an ordinary derivative and the symbol $\partial$ stands for a partial derivative. It is worth noting that the first term in Eq. (6), the derivative $d \sigma_{j} / d X_{i}$, is a derivative related to the direct dependence of $\sigma_{j}$ on $X_{i}$. The second term, 
instead, is an implicit derivative related to the dependence of $\sigma_{j}$ on $X_{i}$ through the nodal displacements $\mathbf{u}$.

The partial derivative $\partial \sigma_{j} / \partial \mathbf{u}$ can be evaluated analytically, or found by finite differences if necessary, since the evaluation of $\sigma_{j}$ when only displacements are changed is straightforward. However, the partial derivative $\partial \mathbf{u} / \partial X_{i}$ is difficult to evaluate, and its computation should be avoided. This can be accomplished by using the adjoint method, as described by Haftka and Gurdal (1992).

\subsection{Adjoint method}

The following system of linear equations relating the applied forces $\mathbf{F}$ and the displacements $\mathbf{u}$ is considered (Bathe 1996):

$$
\mathbf{K} . \mathbf{u}=\mathbf{F},
$$

where $\mathbf{K}$ is the stiffness matrix of the structure. The partial derivative of Eq. (7) with respect to a design variable is

$$
\frac{\partial \mathbf{K}}{\partial X_{i}} \cdot \mathbf{u}+\mathbf{K} \cdot \frac{\partial \mathbf{u}}{\partial X_{i}}-\frac{\partial \mathbf{F}}{\partial X_{i}}=\mathbf{0}
$$

which has been rearranged in order to give an expression equal to a zero vector.

Since Eq. (8) is equal to a zero vector, Eq. (6) can be rewritten as

$$
\frac{\partial g_{j}}{\partial X_{i}}=\frac{d \sigma_{j}}{d X_{i}}+\frac{\partial \sigma_{j}{ }^{T}}{\partial \mathbf{u}} \cdot \frac{\partial \mathbf{u}}{\partial X_{i}}-\boldsymbol{\tau}_{j}{ }^{T} \cdot\left(\frac{\partial \mathbf{K}}{\partial X_{i}} \cdot \mathbf{u}+\mathbf{K} \cdot \frac{\partial \mathbf{u}}{\partial X_{i}}-\frac{\partial \mathbf{F}}{\partial X_{i}}\right)
$$

or

$$
\frac{\partial g_{j}}{\partial X_{i}}=\frac{d \sigma_{j}}{d X_{i}}+\left(\frac{\partial \sigma_{j}{ }^{T}}{\partial \mathbf{u}}-\boldsymbol{\tau}_{j}{ }^{T} \cdot \mathbf{K}\right) \cdot \frac{\partial \mathbf{u}}{\partial X_{i}}-\boldsymbol{\tau}_{j}{ }^{T} \cdot\left(\frac{\partial \mathbf{K}}{\partial X_{i}} \cdot \mathbf{u}-\frac{\partial \mathbf{F}}{\partial X_{i}}\right)
$$


where $\boldsymbol{\tau}_{j}$ is an arbitrary vector. Eq. (10) shows that the evaluation of $\partial \mathbf{u} / \partial X_{i}$ can be avoided if

$$
\frac{\partial \sigma_{j}^{T}}{\partial \mathbf{u}}-\boldsymbol{\tau}_{j}{ }^{T} \cdot \mathbf{K}=\mathbf{0}
$$

Thus, the vector $\boldsymbol{\tau}_{j}$ is chosen as the solution of the system of linear equations

$$
\mathbf{K} . \boldsymbol{\tau}_{j}=\frac{\partial \sigma_{j}}{\partial \mathbf{u}},
$$

where the symmetry of the stiffness matrix has been used (Bathe 1996). Equation (10) then becomes

$$
\frac{\partial g_{j}}{\partial X_{i}}=\frac{d \sigma_{j}}{d X_{i}}-\boldsymbol{\tau}_{j}{ }^{T} \cdot\left(\frac{\partial \mathbf{K}}{\partial X_{i}} \cdot \mathbf{u}-\frac{\partial \mathbf{F}}{\partial X_{i}}\right),
$$

that can be easily evaluated once the vector $\boldsymbol{\tau}_{j}$ is found from Eq. (12). In fact, the derivatives $\partial \mathbf{K} / \partial X_{i}$, and $\partial \mathbf{F} / \partial X_{i}$ can be evaluated analytically (Torii and Biondini 2009), or numerically by finite differences.

It is worth noting that the evaluation of the partial derivative $\partial \mathbf{u} / \partial X_{i}$ could be also avoided by using Eq. (8) rewritten as follows:

$$
\frac{\partial \mathbf{u}}{\partial X_{i}}=\mathbf{K}^{-1} \cdot\left(\frac{\partial \mathbf{F}}{\partial X_{i}}-\frac{\partial \mathbf{K}}{\partial X_{i}} \cdot \mathbf{u}\right)
$$

However, in this approach the number of systems of linear equations to be solved is equal to the number of design variables. In the adjoint method, instead, the number of systems of linear equations is equal to the number of bars in the ground structure. Since the number of design variables is the number of members (cross section areas) plus the number of nodal coordinates taken as design variables, the 
adjoint method will always lead to a lower number of systems of linear equations to be solved.

Finally, it is outlined that if the constraints and the objective function of the optimization problem are both incorporated into a single objective function, by means of the augmented Lagrangean method or some other penalty methods (Rao, 1996), the number of systems of linear equations to be solved during sensitivity analysis can be drastically reduced, as demonstrated by Pereira et al. (2004).

\subsection{Alternative loading conditions}

When a set of $s$ alternative loading conditions $\left\{\mathbf{F}_{1}, \ldots, \mathbf{F}_{k}, \ldots, \mathbf{F}_{s}\right\}$ is considered, the structural response will be defined by a set of nodal displacements $\left\{\mathbf{u}_{1}, \ldots, \mathbf{u}_{k}, \ldots, \mathbf{u}_{s}\right\}$, and the optimization problem from Eq. (4) and Eq. (5) becomes

Find: X

that gives

$$
\min V(\mathbf{X})=\mathbf{A}^{T} \mathbf{. L}
$$

subject to

$$
\mathbf{g}_{k} \leq \mathbf{0} \quad(k=1, \ldots, s),
$$

where the vectors $\mathbf{g}_{k}$ from Eq. (16) are defined for each loading condition in Eq. (2) and Eq. (3).

Working again with constraints related to the yielding stress in compression, since constraints related to tension will just have opposite sign, the derivative of constraint $g_{j k}$, related to the stress in the bar $j$ when the loading condition $k$ is applied, is 


$$
\frac{\partial g_{j k}}{\partial X_{i}}=\frac{d \sigma_{j k}}{d X_{i}}+\frac{\partial \sigma_{j k}^{T}}{\partial \mathbf{u}_{k}} \cdot \frac{\partial \mathbf{u}_{k}}{\partial X_{i}} .
$$

The adjoint vector is now given by the solution of the system of linear equations

$$
\mathbf{K} . \tau_{j k}=\frac{\partial \sigma_{j k}}{\partial \mathbf{u}_{k}}
$$

and Eq. (17) can be rewritten as

$$
\frac{\partial g_{j k}}{\partial X_{i}}=\frac{d \sigma_{j k}}{d X_{i}}-\boldsymbol{\tau}_{j k}{ }^{T} \cdot\left(\frac{\partial \mathbf{K}}{\partial X_{i}} \cdot \mathbf{u}_{k}-\frac{\partial \mathbf{F}_{k}}{\partial X_{i}}\right) .
$$

This formulation leads to an increase in the number of systems of linear equations that must be solved during the sensitivity analysis, i.e. $s$ times the number of systems of linear equations needed for one loading condition. However, it is noted that the stiffness matrix remains the same. Consequently, decomposition techniques can be employed efficiently for the sensitivity analysis, since it may be assumed that the main computational effort lies in the decomposition procedure itself (Bathe 1996).

\subsection{Bounds on the design variables}

There are two different approaches for defining bounds on the nodal coordinates. In the first approach, bounds can be defined locally for each design variable, as shown in Fig. 1a. In this case, there are different bounds for each design variable, and this can be accomplished by defining a rectangular feasible region around each node. The second approach is that of defining bounds for all the design variables at once, as shown in Fig. 1b. In this case, the bounds are the same for all design variables. These two approaches may lead to different results, since the feasible domain defined in the first approach is smaller. However, the first approach may prevent problems related to 
node superposition, if the bounds are defined properly. Therefore, this approach may be recommended when there are many nodes for which the coordinates are taken as design variables in the optimization procedure.

For members areas it is necessary to allow only positive values, defining in this way a lower bound for these design variables. Upper bounds on members areas are not strictly necessary, since the algorithm will seek a structure with minimum volume. However, defining upper bounds on all design variables may be recommended, since the optimization problem becomes better posed.

\section{Probabilistic optimization}

In the following, the deterministic optimization problem presented previously is formulated in probabilistic terms.

\subsection{Formulation of the problem and reliability assessment}

Consider the applied forces $\mathbf{F}$ and the yielding stresses $\sigma_{\text {tor } ~}$ to be random variables with known density distribution. For convenience of notation, they are grouped into the random variable vector $\boldsymbol{\Xi}$. The optimization problem still searches for the minimum volume structure, but now subject to a minimum reliability level of the structure. A component level reliability constraint is considered, instead of dealing with the probabilistic failure constraint at the system level. In other words, a minimum reliability level is enforced for each bar. For a single loading condition this leads to the following problem:

\section{Find: $\mathbf{X}$}

that gives 


$$
\min V(\mathbf{X})=\mathbf{A}^{T} \mathbf{L}
$$

subject to

$$
G_{j}=P_{0}-P\left(g_{j}(\Xi) \leq 0\right) \leq 0 \quad(j=1, \ldots, m),
$$

where $P(\cdot)$ is the probability of the constraint to be fulfilled and $P_{0}$ is a minimum probability level of the constraint or its reliability. Note that now the constraints of the optimization problem are affected by the random variable vector $\boldsymbol{\Xi}$, becoming themselves random variables. The constraint from Eq. (21) states that the probability of $g_{j}(\boldsymbol{\Xi})$ being respected must be higher than a minimum probability $P_{0}$.

The random variable $g_{j}(\boldsymbol{\Xi})$ is constructed based on a linear structural behavior, as occurs in most structural optimization procedures (Hemp, 1973; Pedersen, 1970; Pedersen, 1973; Achtziger, 2006; Achtziger 2007; Martínez et al., 2007; Torii and Biondini, 2009; Pereira et al., 2004), and by assuming $\mathbf{F}$ and $\sigma$ independent normal random variables.

If for a given applied force vector $\mathbf{F}_{0}$, the resulting stress in a given member is $\sigma_{0}$, then for an arbitrary applied force vector $\mathbf{F}$, obtained by the multiplication of $\mathbf{F}_{0}$ by a scalar, the stress in that member is (by the principle of superposition from structural mechanics)

$$
\sigma=\frac{\sigma_{0}}{\left\|\mathbf{F}_{0}\right\|} \cdot\|\mathbf{F}\|=\frac{\sigma_{0}}{F_{0}} \cdot F
$$

where $\|\cdot\|$ denotes the norm of a vector, and $F$ and $F_{0}$ are the norms of $\mathbf{F}$ and $\mathbf{F}_{0}$, respectively. Equation (22) can be substituted into Eq. (2), giving

$$
g(\Xi)=\frac{\sigma_{0}}{F_{0}} \cdot F-\sigma_{t} \leq 0
$$

where the index $j$ has been dropped for convenience. 
Since both the applied forces and yielding stresses are considered as independent normal random variables, a linear combination of them is also a normal random variable. Consequently, by denoting $\mu_{1}$ and $s_{1}$ the mean value and standard deviation of the applied force, respectively, and $\mu_{2}$ and $s_{2}$ the mean value and standard deviation of the yielding stress, respectively, $g(\boldsymbol{\Xi})$ as given by Eq. (23) is a normal random variable with mean

$$
\mu_{g(\Xi)}=\frac{\sigma_{0}}{F_{0}} \cdot \mu_{1}-\mu_{2}
$$

and standard deviation

$$
s_{g(\Xi)}^{2}=\left(\frac{\sigma_{0}}{F_{0}}\right)^{2} \cdot s_{1}^{2}+s_{2}^{2} .
$$

The normalized value of $g(\boldsymbol{\Xi})$ is then:

$$
\beta=\frac{g(\boldsymbol{\Xi})-\mu_{g(\Xi)}}{s_{g(\Xi)}},
$$

where $\beta$ is the reliability or also called reliability index of the constraint (Lemaire et $a l ., 2005)$. Thus, the reliability index is related to the probability of the constraint being feasible by:

$$
\beta=\Phi^{-1}\left[P\left(g_{j}(\boldsymbol{\Xi}) \leq 0\right)\right] \text { or } P\left(g_{j}(\boldsymbol{\Xi}) \leq 0\right)=\Phi[\beta]
$$

where $\Phi=\Phi[\bullet]$ is the standard normal cumulative probability function. The required minimum reliability level of the structure $P_{0}$ is related to the so-called target reliability index by:

$$
P_{0}=\Phi\left[\beta_{t}\right] \text { or } \beta_{t}=\Phi^{-1}\left[P_{0}\right]
$$


Substituting Eq. (27) and (28), the constraint given by Eq. (21) becomes

$$
G_{j}=\beta_{t}-\beta_{j} \leq 0 \quad(j=1, \ldots, m) .
$$

The index $j$ emphasizes that a reliability index is computed for each bar.

\subsection{Sensitivity analysis}

The derivative of Eq. (29) with respect to a design variable is given by

$$
\frac{\partial G_{j}}{\partial X_{i}}=-\frac{d \beta_{j}}{d X_{i}}-\frac{\partial \beta_{j}{ }^{T}}{\partial \mathbf{u}} \cdot \frac{\partial \mathbf{u}}{\partial X_{i}}
$$

where the derivative of the constraint with respect to a given design variable is composed of an explicit plus an implicit derivative, as occurs for the deterministic case. The ordinary derivative $d \beta_{j} / d X_{i}$ can be evaluated by finite differences, since only perturbations to the design variables are necessary. The second part of Eq. (30) can be solved efficiently by applying the adjoint method again, in order to avoid the evaluation of the derivative $\partial \mathbf{u} / \partial X_{i}$. Following the same procedure described for the deterministic approach:

$$
\frac{\partial G_{j}}{\partial X_{i}}=-\frac{d \beta_{j}}{d X_{i}}+\boldsymbol{\tau}_{j}^{T} \cdot\left(\frac{\partial \mathbf{K}}{\partial X_{i}} \cdot \mathbf{u}-\frac{\partial \mathbf{F}}{\partial X_{i}}\right),
$$

where the adjoint vector $\boldsymbol{\tau}_{j}$ is found by solving

$$
\boldsymbol{K} . \boldsymbol{\tau}_{j}=-\frac{\partial \beta_{j}}{\partial \boldsymbol{u}}
$$

The derivative $\partial \beta_{j} / \partial \mathbf{u}$ can be efficiently evaluated by finite differences.

\subsection{Loading conditions given by several forces with different standard deviations}


In order to describe how forces with different standard deviations can be taken into account into the same loading condition, consider the example from Fig. 2. In this case, the structure is subjected to a single loading condition. That is, the forces $\mathbf{F}^{1}$ and $\mathbf{F}^{2}$ are applied at the same time, but have different mean values and standard deviations.

In order to access the reliability of the structure, it is necessary to study the effect of each force, $\mathbf{F}^{1}$ and $\mathbf{F}^{2}$, separately. Thus, considering each force separately (again by the superposition principle)

$$
\sigma^{1}=\frac{\sigma_{0}^{1}}{\left\|\mathbf{F}_{0}^{1}\right\|} \cdot\left\|\mathbf{F}^{1}\right\|=\frac{\sigma_{0}^{1}}{F_{0}^{1}} \cdot F^{1}
$$

and

$$
\sigma^{2}=\frac{\sigma_{0}^{2}}{\left\|\mathbf{F}_{0}^{2}\right\|} \cdot\left\|\mathbf{F}^{2}\right\|=\frac{\sigma_{0}^{2}}{F_{0}^{2}} \cdot F^{2},
$$

where the same notations as of Eq. (22) hold, but here the superscripts 1 and 2 represent the quantities for forces $\mathbf{F}^{1}$ and $\mathbf{F}^{2}$. That is, one structural analysis is made for each force $\mathbf{F}_{0}^{1}$ and $\mathbf{F}_{0}^{2}$, which gives the stresses $\sigma_{0}^{1}$ and $\sigma_{0}^{2}$, respectively (usually, these forces are taken as unit forces, for convenience). The stress caused by the application of $\mathbf{F}^{1}+\mathbf{F}^{2}$ is then

$$
\sigma=\sigma^{1}+\sigma^{2}=\frac{\sigma_{0}^{1}}{F_{0}^{1}} \cdot F^{1}+\frac{\sigma_{0}^{2}}{F_{0}^{2}} \cdot F^{2},
$$

which can be rewritten, for convenience, as

$$
\sigma=k_{1} \cdot F^{1}+k_{2} \cdot F^{2}
$$


The important aspect here is that the effect inside the bar can be written as a linear combination of the separate effects of each force, as this is a linear structural analysis. This is precisely the superposition principle from structural mechanics.

Since the applied forces are Gaussian random variables, the mean value of the stress in the bar and its standard deviation due to the combination of forces $F^{1}$ and $F^{2}$ (Eq. (36)) are, respectively,

$$
\mu_{\sigma}=k_{1} \cdot \mu_{F^{1}}+k_{2} \cdot \mu_{F^{2}}
$$

and

$$
\left(s_{\sigma}\right)^{2}=\left(k_{1} \cdot s_{F^{1}}\right)^{2}+\left(k_{2} \cdot s_{F^{2}}\right)^{2}
$$

where $\mu_{F^{1}}$ and $s_{F^{1}}$ are the mean value and standard deviation of the force $\mathbf{F}^{l}$, respectively (the same notation is also used for the force $\mathbf{F}^{2}$ ). Moreover, $\mu_{\sigma}$ and $s_{\sigma}$ are the mean value and the standard deviation of the stress inside the bar, respectively. Note that the stress is a Gaussian random variable, as it is the linear combination of two Gaussian random variables, namely the two forces.

Thus far it has been shown how to obtain the mean value and the standard deviation of the stress inside the bar, given two forces with magnitude $F^{l}$ and $F^{2}$ acting simultaneously. Consider now the stress constraint from Eq. (2). Since it is a linear combination of Gaussian random variables (the stress and the maximum allowable stress),

$$
\mu_{g(\Xi)}=\mu_{\sigma}-\mu_{\sigma_{t}}
$$

and

$$
s_{g(\Xi)}^{2}=\left(s_{\sigma}\right)^{2}+\left(s_{\sigma_{t}}\right)^{2}
$$


where $\mu_{g(\Xi)}$ and $s_{g(\Xi)}$ are the mean and the standard deviation of the constraint, and $\mu_{\sigma_{t}}$ and $s_{\sigma_{t}}$ are the mean and the standard deviation of the maximum allowable stress in tension.

It is possible to evaluate Eq. (39) and Eq. (40) with Eq. (37) and Eq. (38), and consequently, to obtain the reliability index as described previously. Also, note that Eq. (39) and Eq. (40) are analogous to Eq. (24) and Eq. (25), respectively.

The previous results can also be extended to more than two forces acting at the same time. However, this leads to a significant increase in the number of structural analysis that must be made. Also, the sensitivity analysis is also changed, since now there will be one adjoint vector associated with each force.

\section{Numerical examples}

In this section, several numerical examples are solved in order to discuss the main aspects of the approach presented. Note that for all the following examples each figure has its own scale, and the reader can compare the different solutions by the volume of material $V$ presented for each example. A lower bound for the areas is defined as $1 \mathrm{E}-10 \mathrm{~m}^{2}\left(\right.$ i.e. $\left.0.1 \mathrm{~mm}^{2}\right)$, in order to avoid singularity of the stiffness matrix. Finally, the optimization algorithm used here is the Sequential Quadratic Programming (SQP) (Nocedal and Wright, 1999; Rao, 1996).

\subsection{Example 1 - two bar structure}

The first example discussed here is the optimization of a two bar structure. Even if this example is simple from the practical point of view, it demonstrates what changes may arise when the yielding stresses have different standard deviations. 
The ground structure from Fig. 3a is subjected to a single loading condition with mean $F=10,000 \mathrm{~N}$ and standard deviation $\sigma_{F}=1,000 \mathrm{~N}$. The lengths of the structure are $L_{x}=2.5 \mathrm{~m}$ and $L_{y}=5 \mathrm{~m}$ and the Young Modulus of the material is $E=$ 200GPa. The minimum reliability index is taken as $\beta=3.1$. The yielding stress in tension has mean $\sigma_{t}=+250 \mathrm{MPa}$ and the yielding stress in compression has mean $\sigma_{c}=$ -250MPa. For the case of Fig. 3c the yielding stresses in tension and in compression have standard deviation $\sigma_{\sigma}=10 \mathrm{MPa}$; while for the case of Fig. $3 \mathrm{~d}$ the standard deviation of the yielding stress in tension is raised to $\sigma_{\sigma t}=50 \mathrm{MPa}$. The node of the applied forces is allowed to be moved up and down by the optimization algorithm.

From the results presented in Fig. 3 it can be seen that changing only the standard deviation of the yielding stresses leads to changes in the topology and geometry of the optimum structure. The structure in Fig. $3 \mathrm{c}$ is symmetric, since the yielding stresses both in tension and compression have the same mean value, in magnitude, and the same standard deviation. The solution of the deterministic problem, from Fig. 3b, is also symmetric. However, the structure from Fig. 3d is not symmetric because of the different values of the standard deviation of the yielding stresses. Finally, note that increasing the standard deviation of the yielding stress leads to an increase in the volume of the structure, as expected.

\subsection{Example 2 - Effect of the reliability index}

The ground structure from Fig. $4 \mathrm{a}$ has lengths $L_{x}=9 \mathrm{~m}$ and $L_{y}=1 \mathrm{~m}$. The material properties are the same as the ones defined for the example 1. The structure is subjected to 8 loading conditions with mean $F=1,000 \mathrm{~N}$ and standard deviation $\sigma_{F}=$ $100 \mathrm{~N}$. Three cases are studied, the deterministic problem, the problem for $\beta$ equal to 3.1 and for $\beta$ equal to 4.75 . The nodes of the upper chord are allowed to be move up 
and down by the optimization algorithm, to positions as far as $0.9 \mathrm{~m}$ from the original position.

For the three different cases the optimum topology and the geometry remain the same. The only difference is that all bar areas are scaled, and the volume of material increases for increasing reliability indexes. From this example, one may conclude that increasing the reliability index will always lead to a scaled solution, but this is not true for all cases, as demonstrated by the next example.

\subsection{Example 3 - Effect of the reliability index}

In order to show that increasing the reliability index may lead to geometry and topology changes, return to the example from Fig. 3. The loads and dimensions of the ground structure remain unchanged as well as most of the material properties. The only modification is that the standard deviation of the yielding stress in compression is now equal to $\sigma_{\sigma c}=10 \mathrm{MPa}$ and of the yielding stress in tension equal to $\sigma_{\sigma t}=$ $20 \mathrm{MPa}$. The problem is then solved for reliability indexes equal to 0 (deterministic problem), 2, 4, 6, 8 and 10. The results are presented in Fig. 5. It can be seen that increasing the reliability index leads, in this case, to changes in both topology and geometry. This example contradicts the assumption of only scaling the cross-section areas when different reliability indexes are used.

The reason why the example from Fig. 4 gave scaled solutions for different reliability indexes lie in the fact that all the properties of that ground structure are symmetric. The mean values and standard deviation of the yielding stresses are equal in magnitude, together with the applied forces. The case from Fig. 5, instead, has different standard deviations for the yielding stresses, and this leads to changes in topology and geometry when the reliability index is changed. 


\subsection{Example 4-Effect of limiting the maximum area of the bars}

The ground structure of this example is shown in Fig. 6, and this example demonstrates the effect of defining a maximum value for the bar areas. The structure lengths are $L_{x}=2 \mathrm{~m}$ and $L_{y}=1 \mathrm{~m}$. The material properties are the same as the ones defined for the example 1 . The structure is subjected to one loading condition with mean value $F=1,000 \mathrm{~N}$ and standard deviation $\sigma_{F}=100 \mathrm{~N}$. The nodes are taken as fixed during the optimization procedure, and thus only sizing optimization is needed for this case. Additionally, for this problem the bar areas are not allowed to be bigger than $1 \mathrm{E}-8 \mathrm{~m}^{2}$ (i.e. $10 \mathrm{~mm}^{2}$ ). Three cases are studied, with $\beta$ equal to 0 (deterministic problem), 3.1 and 4.75 .

The results from Fig. 6 show that defining a maximum bar area may lead to different topologies when only the reliability index is changed. This happens since the maximum bar area may enforce the optimization algorithm to "use" more bars in order to increase the reliability of the structure, leading to different topologies. Besides, it is reasonable to assume that different topologies will lead to different geometries if geometry optimization is pursued.

\subsection{Example 5 - Loading conditions composed of several forces}

Figure 7 presents a ground structure that is subjected to three loading conditions. However, vertical forces and horizontal forces have different mean values and standard deviations. This example illustrates the application of the method when a loading condition is composed by forces with different mean values and standard deviations. 
The ground structure from Fig. 7 has a total height of $4 \mathrm{~m}$ and a total width of $2 \mathrm{~m}$. The material properties are the same as from example 1 . The target reliability index is 3.1, and all nodes (except the nodes of the supports) are allowed to be moved left and right by the optimization algorithm, to positions as far as $0.8 \mathrm{~m}$ from its original position. The vertical force mean value is $F_{1}=1,000 \mathrm{~N}$ and its standard deviation is $\sigma_{F 1}=100 \mathrm{~N}$. For the horizontal forces $F_{2}$, three cases are studied: mean equal $F_{2}=50 \mathrm{~N}$ and standard deviation $\sigma_{F 2}=5 \mathrm{~N}$; mean $F_{2}=150 \mathrm{~N}$ and standard deviation $\sigma_{F 2}=15 \mathrm{~N}$; and mean $F_{2}=250 \mathrm{~N}$ and standard deviation $\sigma_{F 2}=25 \mathrm{~N}$. Finally, symmetry of the geometry is enforced.

The results for this example are presented in Fig. 8. As a consequence of the increase to the lateral load, for the same vertical load, there was an increase of the volume of the structure and a change of its geometry and topology. The structures designed for higher lateral loads are clearly more fitted to resisting the increased bending moment that develops in these cases.

For the same ground structure from Fig. 7, another interesting example can be conceived. Taking the vertical force $F_{1}$ as defined previously and the mean of the horizontal force $F_{2}=250 \mathrm{~N}$, the same problem is now solved for three different standard deviations: $\sigma_{F 2}=25 \mathrm{~N}, \sigma_{F 2}=50 \mathrm{~N}$ and $\sigma_{F 2}=75 \mathrm{~N}$. The results are presented in Fig. 9, where it can be noted that increasing the standard deviation of the lateral load leads to an increase in the volume of the structure. Also, the geometry and the topology change in order to resist the increased bending moments that appear in this case.

\subsection{Example 6-Comparison of the sizing and simultaneous sizing-geometry optimization}


The example from Fig. 10 compares results from the simultaneous optimization of geometry and sizing with results given by sizing optimization only. The ground structure has a total height of $1 \mathrm{~m}$ and a total length of $8 \mathrm{~m}$. The material properties are as defined for the example 1 . The target reliability index of the structure is $\beta=4.75$, and all the nodes from the upper chord are allowed to be moved up and down, by the optimization algorithm, to positions as far as $0.5 \mathrm{~m}$ from its original position. The applied force is $F=1,000 \mathrm{~N}$ with a standard deviation $\sigma_{F}=100 \mathrm{~N}$.

The results from Fig. 10 demonstrate that changes in the geometry of the structure can lead to significant improvements of the solution, even if the topology (the bars that comprise the structure) remains basically the same. This justifies the effort in including the nodal coordinates as design variables of the optimization problem.

\subsection{Example 7 - Local minima}

The last example is used to illustrate the existence of local minima that can occur for problems of truss optimization. For this task, the ground structure from the example of Fig. 6a is used, with $\beta$ equal to 4.75 , but the maximum cross section area is taken as $5.0 \mathrm{E}-7 \mathrm{~m}^{2}\left(\right.$ i.e. $\left.500 \mathrm{~mm}^{2}\right)$. In this case the upper bound for the cross section area does not affect the results. Also, the optimization algorithm is now allowed to move the nodes in the upper chord (except the nodes of support) up and down to positions as far as $0.5 \mathrm{~m}$ from its original positions. This optimization problem is solved several times with random initial solutions. Two different local optima found for this problem are presented in Fig. 11. Note that the existence of local optima is also common for the deterministic case (Achtziger, 2006; Achtziger, 2007; Kocvara and Zowe; 1996). 
For the examples presented earlier, the initial bar areas were all taken equal to $1 \mathrm{E}-9 \mathrm{~m}^{2}\left(\right.$ i.e. $\left.1 \mathrm{~mm}^{2}\right)$, since it was observed that starting with solutions for which the constraints were not respected gave, in general, better results. This can be explained by the following reasoning. If the initial solution is over-designed, then the algorithm will try to reduce the member lengths in order to reduce the volume of the structure, and will not "care" about stress constraints since the current solution is over-designed. This may bring the solution to a geometry composed of shorter members, but which may not be the best geometry possible. However, when starting at solutions for which the constraints are violated, the algorithm will try to rearrange the geometry of the structure in order to reduce the stresses in the bars. This will probably bring the solution to a "good" geometry that was obtained with the intent of reducing the stresses inside the structure, instead of just trying to obtain shorter bars.

\subsection{Further Comments}

Note that the optimization problems presented in this article have several reliability constraints, and consequently several reliability analysis problems must be solved in order to evaluate a given design point of the optimization process. Here, all these reliability analysis problems are solved analytically, and consequently the main computational effort lies in finding the stresses inside the structures for each applied load, i.e. one needs to solve one system of linear equations for each applied load. If the reliability analysis problems were solved using a traditional approach (e.g. FORM, Monte Carlo Simulation), an entire reliability analysis problem would need to be solved for each constraint. It is well known that solving a reliability analysis problem using these approaches leads to the solution of several systems of linear 
equations (since each constraint must be evaluated several times to obtain each reliability index), which leads to an increased computational effort. Take for example the structure from Fig. 7. In this case, there are 21 bars and 3 loading conditions. Since there are 2 constraints for each bar for each loading condition, the optimization problem has a total of $21 \times 2 \times 3=126$ reliability constraints. In a traditional approach (e.g. FORM), one would need to solve 126 reliability analysis problems in order to evaluate a given design point, and each reliability analysis problem would need to solve several systems of linear equations. However, if the approach presented in this article is employed, one only needs to find the stresses inside the structure for each of the 10 loads of this problem ( 2 from loading condition 1, 4 from loading condition 2 and 4 for loading condition 3), i.e. one needs to solve 10 systems of linear equations in order to evaluate a given design point and the remaining information (the reliability index of each constraint) is evaluated analytically. Thus, the computational effort involved in the present approach is orders of magnitude smaller than if a FORM algorithm were used. Regarding stability, the extension of the proposed approach to consider Eulerian buckling is straightforward. The Eulerian buckling limit stress depends only on the moment of inertia, the length and the Young's Modulus of each bar and consequently does not depend on the random variables considered in this article (yielding stress and applied forces). That is, in a Eulerian buckling limit state function, only the buckling stress on the bar would be a random variable, different from the yielding stress case (Eq.(3)) where both the stress on the bar and the yielding stress are random variables. Thus, the inclusion of a buckling constraint would be made by the same procedure as for a standard compression stress constraint, but by taking the allowable buckling stress as a deterministic value. 


\section{Conclusions}

This article presented a formulation for the simultaneous optimization of topology and geometry of truss structures. General aspects such as constraints and several loading conditions were also discussed, together with a detailed discussion on sensitivity analysis.

The deterministic optimization scheme was then extended to the case when the yielding stresses and the applied forces are Gaussian random variables. The important aspect here is that considering these variables as Gaussian allows one to access the reliability of the structure directly, without using iterative methods such as a First Order Reliability Method (FORM) or Monte Carlo Simulation (MCS). Also, the sensitivity analysis is also simplified by the use of the adjoint method. Thus, the probabilistic problem as defined here has a similar computational cost per optimization step as the deterministic problem, and that is an important aspect.

The main drawbacks of the proposed approach are: (i) not considering the bar areas and the Young Modulus as random variables; (ii) modeling the applied forces and the yielding stresses as Gaussian random variables. However, this decision is based on three main aspects.

First, the resulting optimization problem is simplified and the computational effort greatly reduced. Reliability Based Optimization problems often lead to a significant increase in computational effort, and may be even unaffordable from the computational point of view. Consequently, to reduce the computation effort is a main objective of this article.

Second, the cross-section areas are, in most cases, to be chosen from an array of discrete values. Consequently, the bar areas are expected to be rounded up to the nearest standard area available in the market. In this context, considering the bar areas 


\section{References}

Achtziger W (2006) Simultaneous optimization of truss topology and geometry, revisited. In: Bendsøe M, Olhoff N, Sigmund O (eds) IUTAM Symposium on topological design optimization of structures, machines and materials: status and perspectives. Springer, Berlin Heidelberg New York, pp 413-423

Achtziger W (2007) On simultaneous optimization of truss geometry and topology. Struct Multidisc Optim 33:285-305

Arora JS (2004) Introduction to optimum design, Elsevier, San Diego

Bathe KJ (1996) Finite element procedures. Prentice Hall, Englewood-Cliffs 
Beyer HG, Sendhoff B (2006) Robust optimization - a comprehensive review. Comput Methods Appl Mech Engrg 196:3190-3218

Calafiore GC, Dabbene F (2008) Optimization under uncertainty with applications to design of truss structures. Struct Multidisc Optim 35:189-200

Haftka RT, Gurdal Z (1992) Elements of structural optimization. Kluwer, Dordrecht

Haldar A, Mahadevan S (2000) Reliability assessment using stochastic finite element analysis. John Wiley, New York

Kocvara M, Zowe J (1996) How mathematics can help in design of mechanical structures. In Griffiths DF and Watson GA Watson (eds) Numerical Analysis. Longman, Harlow, pp 76-93.

Lagarosa ND, Plevris V, Papadrakakis M (2007) Reliability based robust design optimization of steel structures. Int J Simul Multidisci Des Optim 1:19-29

Madsen HO, Krenk S, Lind NC (1986) Methods of structural safety. Prentice Hall, Englewood Cliffs

Martínez P, Martí P, Querin OM (2007) Growth method for size, topology, and geometry optimization of truss structures. Struct Multidisc Optim 33:13-26

Murotsu Y, Shao S (1990) Optimum shape design of truss structures based on reliability. Struct Optim 2:65-76

Nakib R (1997) Deterministic and reliability-based optimization of truss bridges. Comput Struct, 65(5):767-775

Nocedal J, Wright SJ (1999) Numerical optimization. Springer-Verlag, Berlin

Hemp WS (1973) Optimum structures. Clarendon, Oxford

Pedersen P (1970) On the minimum mass layout of trusses. Symposium on structural optimization. AGARD Conf Proc 36:189-192

Pedersen P (1993) Topology optimization of three-dimensional trusses. In: Bendsøe MP, Mota Soares CA (eds) Topology design of structures. Kluwer, Dordrecht, pp 19-30

Pereira JT, Fancello EA, Barcellos CS (2004) Topology Optimization of continuum structures with material failure constraints. Struct Multidisc Optim 26:50-66

Rao SS (1996) Engineering optimization - theory and practice. John Wiley, New York

Schuëller GI, Jensen HA (2009) Computational methods in optimization considering uncertainties - an overview. Comput Methods Appl Mech Engrg 198:2-13

Stocki R, Kolanek K, Jendo S, Kleiber M (2001) Study on discrete optimization techniques in reliability-based optimization of truss structures. Comput Struct 79:2235-2247

Thampan CK, Krishnamoorthy CS (2001) System reliability-based configuration optimization of trusses. J Struct Engrg 27(8):947-956

Togan V, Daloglu, A (2006) Reliability and reliability-based design optimization. Turkish J Eng Env Sci 30:237-249

Torii AJ, Biondini F (2009) A simple geometry optimization method for statically indeterminate trusses. 8th World Congress on Structural and Multidisciplinary Optimization, Lisbon 


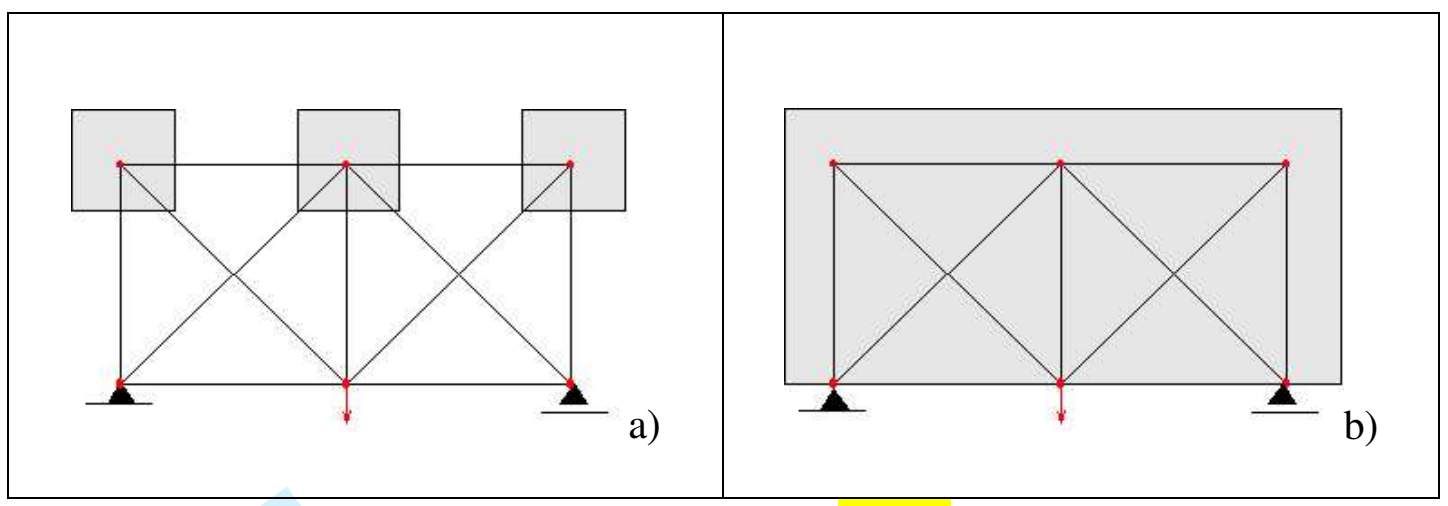

Figure 1: Bounds on nodal coordinates defined a) locally for each node, and b) globally for all nodes. 


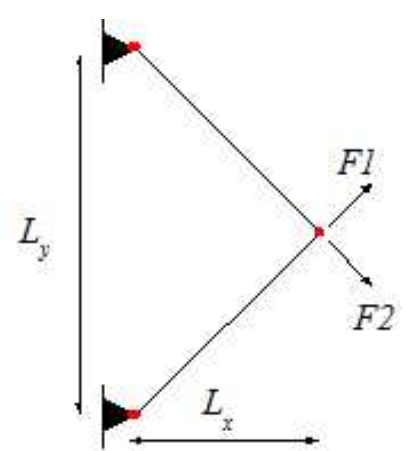

Figure 2: Example of a structure subject to one loading condition given by two forces with different standard deviations. 


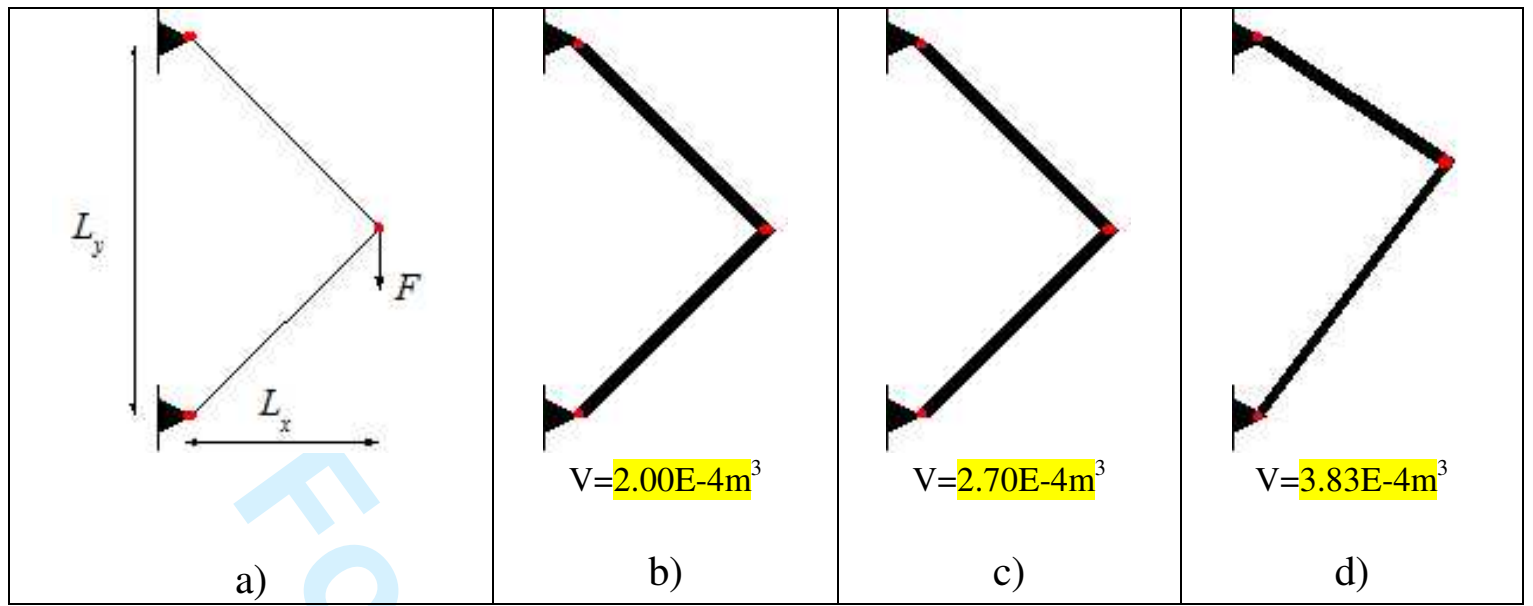

Figure 3: a) Ground structure, b) optimum solution for the deterministic problem, c) optimum solution with yielding stresses in tension and compression with the same standard deviation and d) optimum solution with yielding stress in tension with a higher standard deviation. 


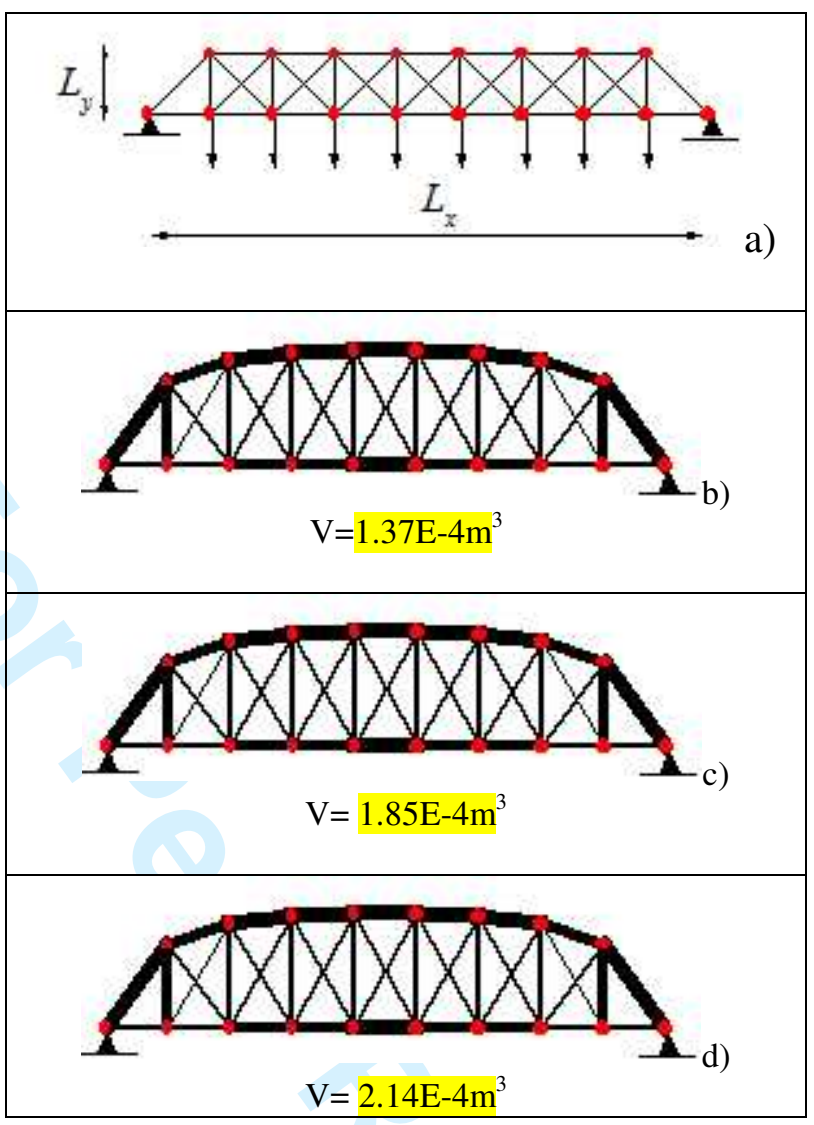

Figure 4: a) Ground structure and optimum solutions b) deterministic problem, c) $\beta$ equal to 3.1 and d) $\beta$ equal to 4.75 . 


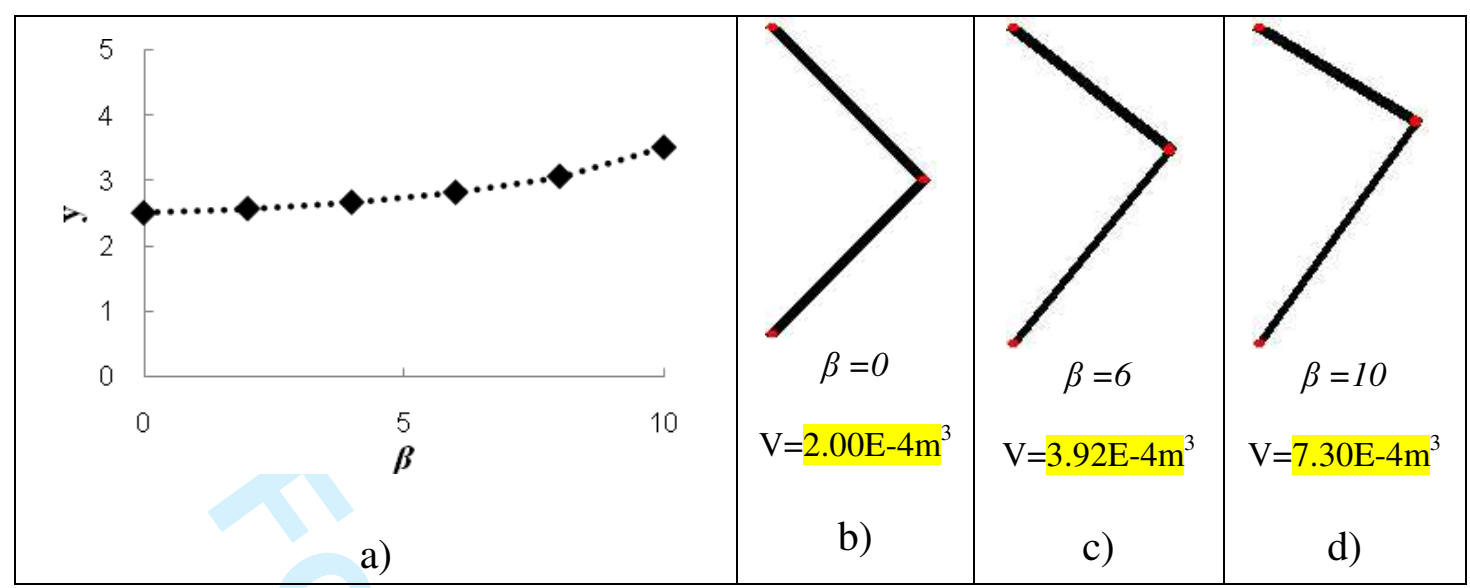

Figure 5: a) The change of the $y$ coordinate of the central node of the optimal design for different reliability indexes and b) c) d) some chosen optimum solutions. 


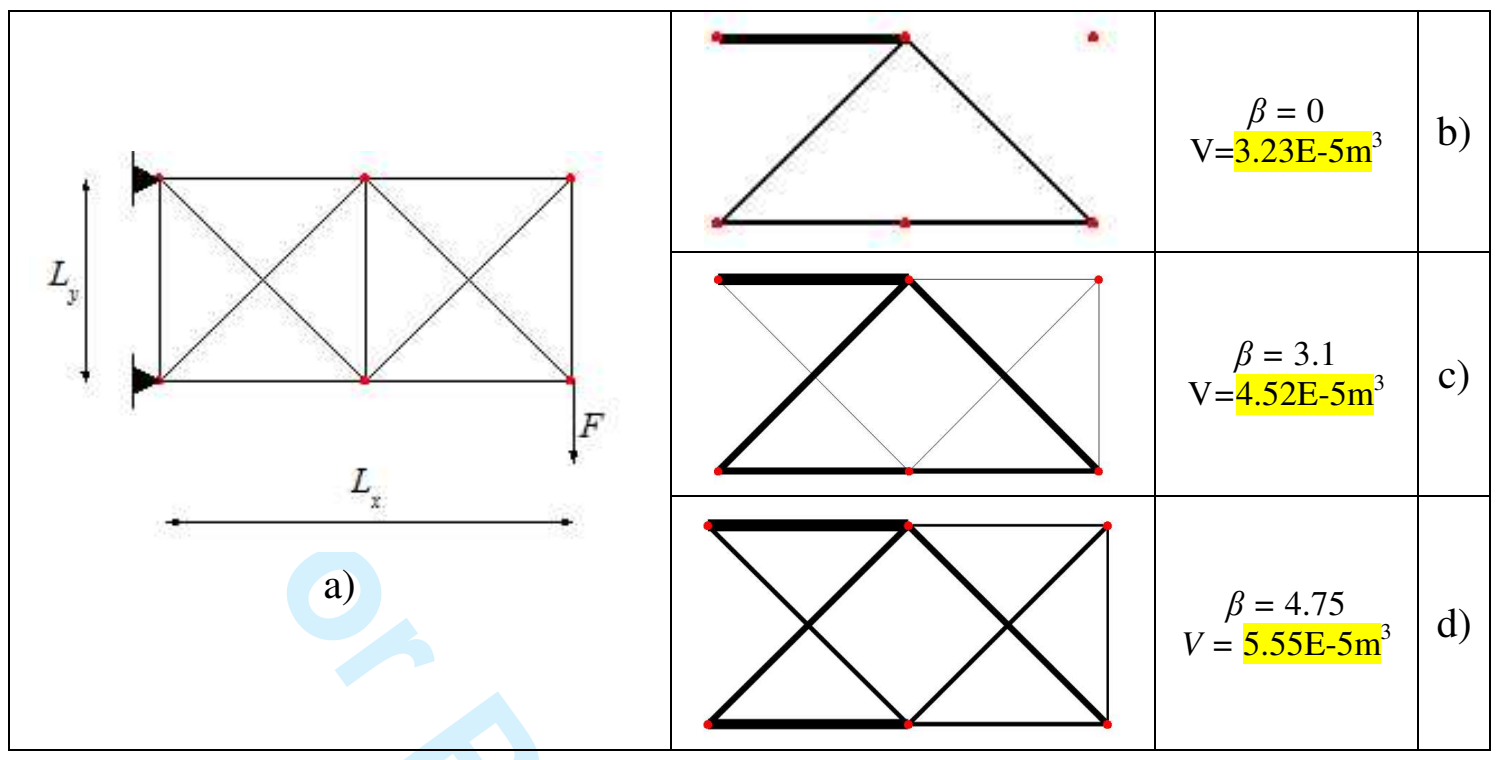

Figure 6: a) Ground structure and b) c) d) optimum topology for different reliability indexes when a maximum cross section area is defined. 


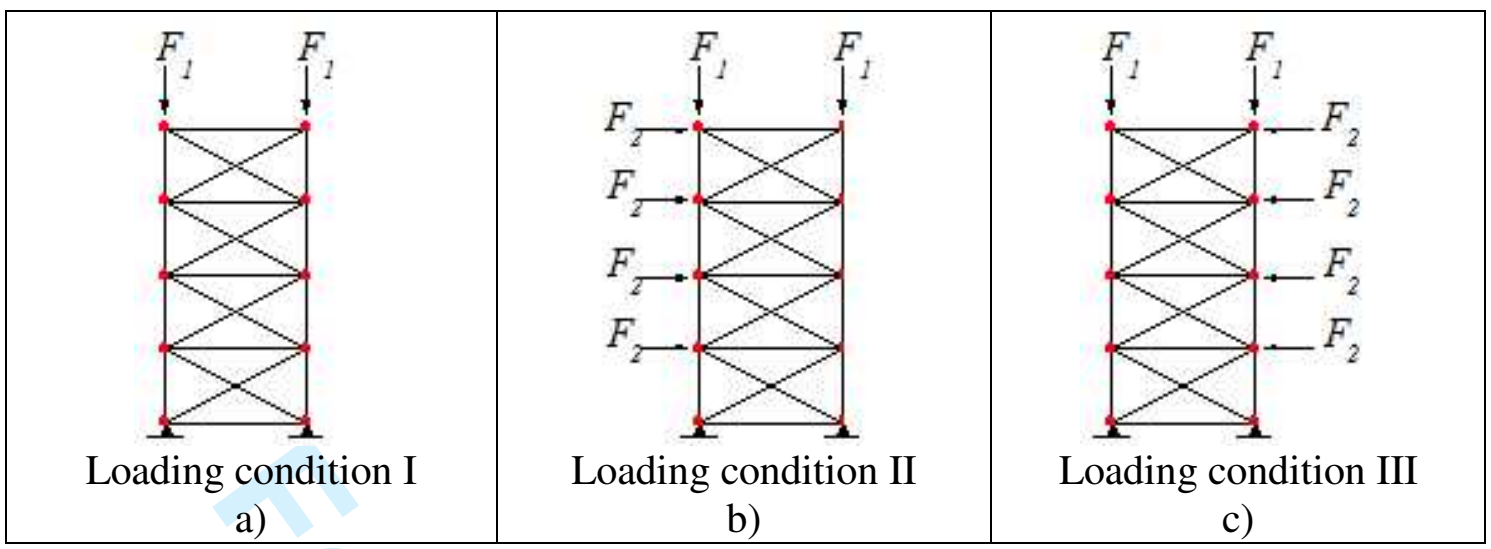

Figure 7: Ground structure subjected to three loading conditions I, II and III. 
$\Delta \bar{\Delta} \Delta$ 
$\overline{A B}$ 


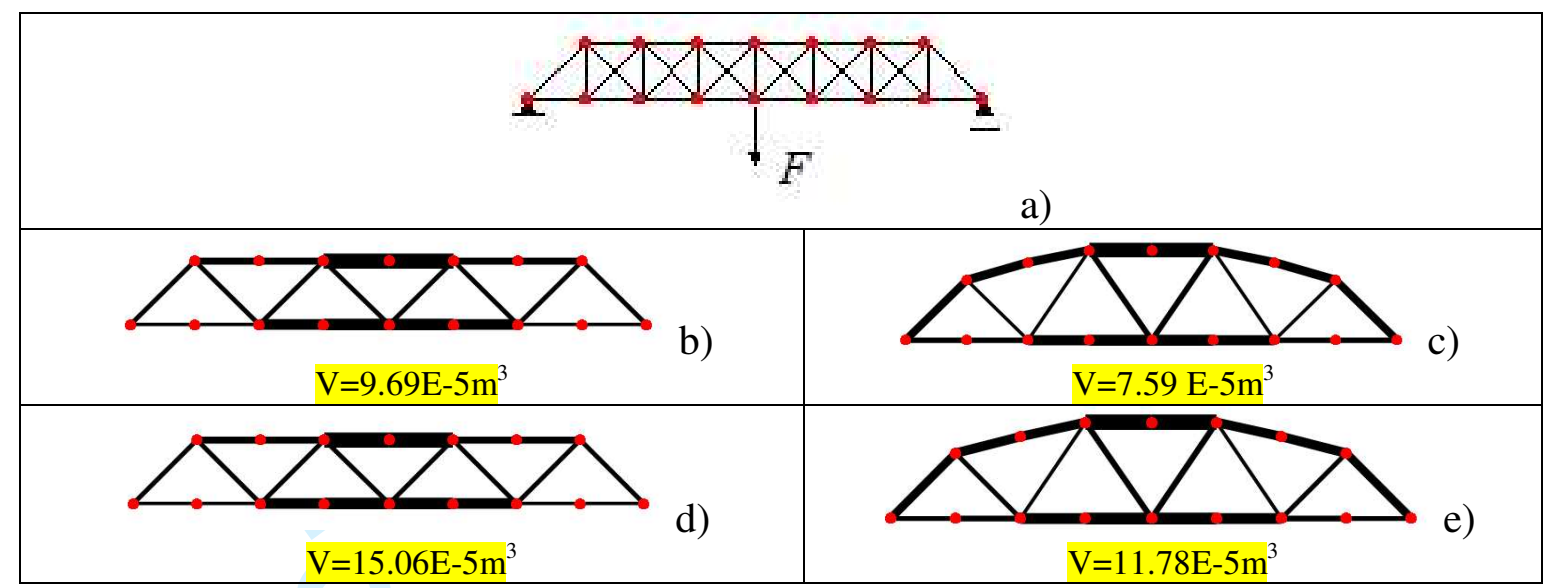

Figure 10: a) Ground structure, b) optimum topology for the deterministic problem, c) optimum geometry for the deterministic problem, d) optimum topology for the probabilistic problem, e) optimum geometry for the probabilistic problem. 


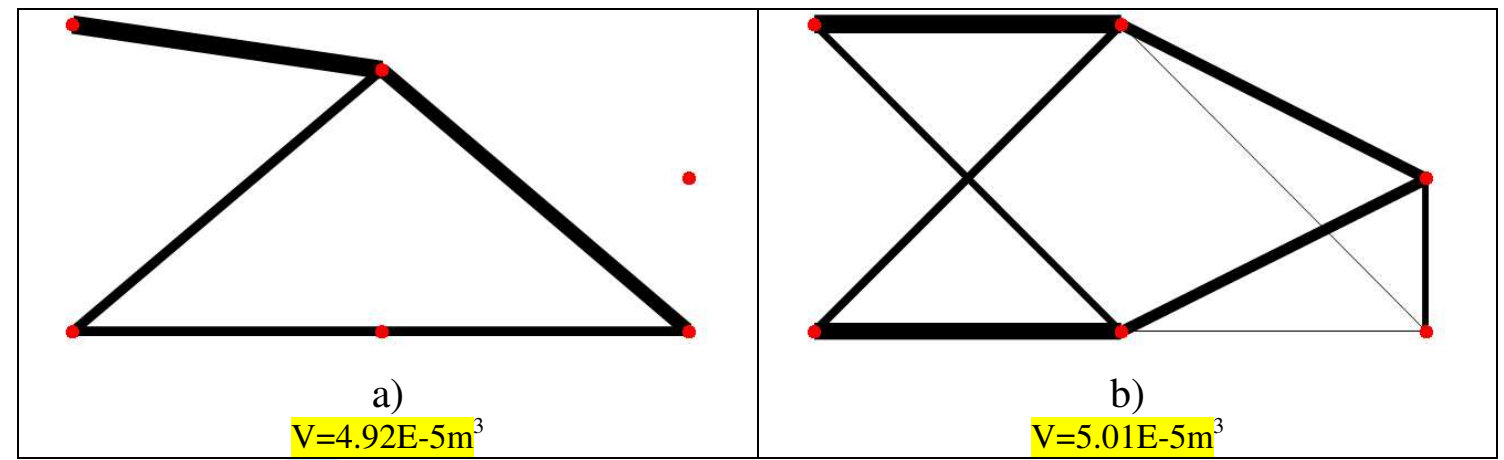

Figure 11: Two different local optima for the probabilistic problem. 